\title{
NEW YORK TIMES TOPICS, UN MODELO DE PRODUCTO PERIODISTICO DOCUMENTAL EN LA PRENSA DIGITAL
}

\author{
Pere Franch y Javier Guallar
}
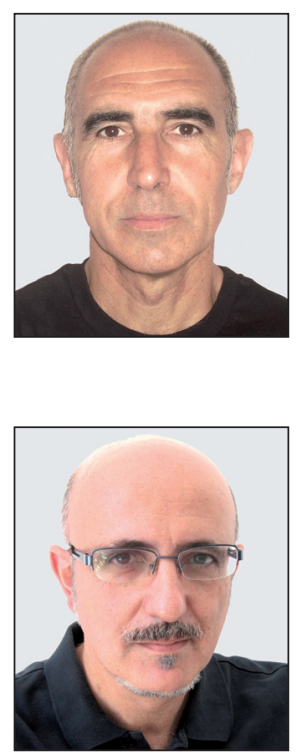

Pere Franch es profesor de periodismo y de relaciones internacionales en la Facultad de Comunicación y Relaciones Internacionales Blanquerna de la Universitat Ramon Llull (URL), donde es también coordinador del Departamento de Periodismo. Ha trabajado como periodista en radio (COM Radio) y prensa escrita (El observador, y Avui, diario para el que fue corresponsal en Estados Unidos). Especialista en información internacional. Mantiene el blog Made in America en el diario Ara.

Universitat Ramon Llull. Facultat de Comunicació Blanquerna Valdonzella, 23. 08001 Barcelona, España http://blogspersonals.ara.cat/madeinamerica PereFP@blanquerna.url.edu

Javier Guallar es profesor en la Facultad de Biblioteconomía y Documentación de la Universitat de Barcelona (UB) y en la Facultad de Comunicación Blanquerna de la Universitat Ramon Lull (URL), y colaborador docente en los Estudios de Información y Comunicación de la Universitat Oberta de Catalunya (UOC). Es subdirector de la revista El profesional de la información, coordinador del Anuario ThinkEPI y codirector de la colección de libros El profesional de la información (Editorial UOC). Anteriormente ha trabajado como documentalista en varios diarios.

Universitat de Barcelona. Facultat de Biblioteconomia i Documentació Melcior de Palau, 140. 08014 Barcelona, España jguallar@gmail.com

\section{Resumen}

Se presentan los productos periodísticos documentales de la prensa digital denominados Temas y Especiales, y tras una revisión bibliográfica, se realiza un estudio de caso de Times topics del diario The New York times. El método empleado es la observación de la sección Topics a lo largo de varios meses de 2011, complementado con una entrevista a su principal responsable, John O’Neil. Se analizan cuestiones como su origen y objetivos, método de trabajo, tipos de páginas de Topics, estructura, análisis de los elementos constituyentes de la página de la sección y análisis de los elementos de la página de cada tema. Se concluye señalando sus principales aportaciones y sus aspectos mejorables.

\section{Palabras clave}

Prensa digital, Periodismo digital, Documentación periodística, Especiales, Temas, The New York times, Times topics.

\section{Title: New York Times Topics, a model of journalistic documentation product in digital press}

\begin{abstract}
After introducing journalistic documentation products in digital media such as Special reports and Topics, this paper reviews the literature about this issue and presents a case study of The New York Times Topics section. The method followed comprises the study of the site over several months during 2011, complemented with an interview with its current chief editor, John O'Neil. Issues such as the origin and objectives of the section, work method, types of Topics pages, page structure, and constituent elements of the section and particular pages are discussed. The main contributions of The New York Times Topics and some areas for improvement are pointed.
\end{abstract}

\section{Keywords}

Digital media, Digital journalism, Journalistic documentation, News librarianship, Special reports, Topics, The New York Times, Times Topics.

Franch, Pere; Guallar, Javier. "New York times topics, un modelo de producto periodístico documental en la prensa digital". El profesional de la información, 2012, septiembre-octubre, v. 21, n. 5, pp. 482-490. 


\section{Introducción}

Temas (o Topics) y Especiales (o Special reports) son un tipo de productos característicos de la prensa digital, que no existen en la prensa impresa, aun cuando se puedan ver antecedentes de los mismos en los grandes reportajes. No suelen ser habituales en diarios modestos o de alcance local o regional, pero sí los podemos ver en muchas de las grandes cabeceras de la prensa digital.

Ofrecen información en profundidad sobre temas, personajes o acontecimientos, con una notable aportación de los elementos propios y diferenciadores de la información periodística digital, como la hipertextualidad o la multimedialidad, y destacando entre ellos la intervención de la documentación. Rubio-Lacoba (2007, p. 126) los define como "productos documentales elaborados específicamente para explicar o indagar con mayor detalle en las noticias más relevantes". Se pueden considerar por ello productos periodísticos documentales.

Temas y Especiales ofrecen información en profundidad sobre temas, personajes o acontecimientos, con una notable aportación de documentación

A pesar de su relevancia en el periodismo digital actual, no han sido objeto de una especial atención por parte de la investigación especializada en periodismo o en documentación. Además, creemos necesario reclamar la atención y el estudio sobre este tipo de productos desde el punto de vista de la documentación, incorporándolos a los temas habituales de la bibliografía sobre documentación en los cibermedios, como pueden ser el estudio de las tareas documentales en los centros especializados (Sánchez-Vigil; Marcos-Recio; Olivera-Zaldua, 2009; Guallar, 2011a) o el análisis de hemerotecas, buscadores y sistemas de recuperación de la información de los diarios (Martínez-Rubio, 2008; Guallar; Rovira; Ruiz, 2010; Rubio Lacoba; Blanco-García, 2010).

En este trabajo se contribuye a su análisis y conocimiento a partir del estudio de caso de un producto de referencia: Times topics del diario The New York times.

\section{Objetivo y método}

El objetivo es analizar las características del producto informativo documental que bajo la denominación Times topics ofrece la edición online del diario The New York times. El objeto de análisis seleccionado se puede considerar representativo de los productos de la prensa digital denominados Temas (Topics) o Especiales (Special reports) ya que The New York times es un diario de referencia mundial y esta sección del diario es uno de los ejemplos más destacados y valorados de este tipo de productos.

El método seguido ha sido la observación directa de la página Times topics y el análisis de su contenido, complementado con una entrevista por correo electrónico a su máximo responsable, John O’Neil.
Se han analizado los dos niveles en los que se presenta: la página principal de la sección y las páginas de cada tema. La observación directa se ha realizado en varias fases a lo largo de 2011, de abril a diciembre, y la entrevista se realizó el 2 de abril de 2011.

Los elementos de análisis que se han considerado son los siguientes:

- origen y objetivos de la sección

- método de trabajo

- tipos de páginas de topics

- autoría

- interacción con el usuario, contacto, página de ayuda

- estructura de las páginas: distribución en columnas, número de elementos constituyentes, ubicación en la página

- análisis de los elementos de la página de la sección

- análisis de los elementos de la página de cada tema

\section{Antecedentes}

Como se ha señalado antes, Temas y Especiales no han gozado de una gran atención por parte de la bibliografía especializada, si bien en España los Especiales en concreto sí han merecido algo más de interés, quizá porque están más extendidos en los diarios digitales españoles. A continuación se comentan algunas de las aportaciones a su estudio que se pueden encontrar en la bibliografía.

Nuño (2003) analiza la estructura del sitio web de 6 diarios españoles, y en el período estudiado (septiembre-octubre 2002) constata la existencia de los documentos denominados Especiales, que define como "auténticos dossiers aglutinando informaciones de distinta naturaleza (fotoperiodística, audiovisual, sonora, textual)..., que no se centra exclusivamente en noticias de actualidad". Domingo (2005), a partir de un estudio etnográfico realizado en 2003 en redacciones de varios medios, hace una valoración elogiosa de estos productos al considerarlos una excepción en la rutina del periodismo digital acuciado por la presión de la actualización constante, ya que, sin esa presión, permiten experimentar y acercarse a un ideal de periodismo digital de calidad. Por su parte, Albornoz (2006) considera los documentos o secciones especiales como "la punta de lanza de la experimentación multimedia".

Rubio-Lacoba (2007, pp. 130-135) analiza extensamente estos productos, que encuadra en lo que denomina "documentación en la información periodística atemporal", y distingue dos tipos: Especiales y Temas. Los primeros poseen una mayor vinculación con la agenda mediática, puesto que suelen elaborarse con motivo de algún acontecimiento cíclico, de vigencia temporal (grandes certámenes deportivos, giras musicales...). Los Temas son productos documentales que abordan los grandes asuntos de reflexión general, de permanente actualidad y de constante actualización (por ejemplo, en el caso de la prensa española, son temas recurrentes la inmigración o el terrorismo). Rubio-Lacoba señala cuatro características de los Temas, que, como veremos, son aplicables a los Topics de The New York times. Son productos documentales:

- con inicio, pero sin final determinado; 
- de diseño uniforme, a partir de una plantilla que se repite con mínimas variaciones;

- de actualización constante; y

- su elaboración es por proceso acumulativo.

Otras aportaciones en la bibliografía de los últimos años son las de Larrondo-Ureta $(2007,2008)$, que analiza en detalle los Especiales de tres diarios españoles; López-Aguirre (2009), que incluye Temas y Especiales dentro de la clasificación de productos documentales que utiliza en su estudio de la prensa digital mexicana; y Guallar (2011b), en su repertorio de productos documentales de la prensa digital. En este último texto se indica:

"La diferencia principal entre los Especiales (Special reports) y los Temas (Topics) es que los primeros suelen estar más vinculados a un hecho noticioso puntual y no se actualizan una vez realizados (se incluyen aquí los clásicos Resúmenes del año), mientras que los segundos son atemporales, desarrollan en profundidad un tema y se siguen actualizando. Su estructura y composición es muy diversa, así como el nivel de participación del departamento de Documentación, dependiendo de la empresa periodística. Se pueden señalar en España las secciones de Especiales de El mundo y $A b c$, y de Temas (denominada "A fondo") de El país, y a nivel internacional, destaca la magnitud de los Topics de The New York times" (Guallar, 2011b).

Asimismo existen 2 trabajos recientes de interés realizados en Estados Unidos sobre Times topics en concreto: una entrevista a John O'Neil (Beckett, 2011) y un estudio de usuarios mediante la técnica de eye tracking (Paul; Hansen; Suzuki, 2011). En este último se muestra la valoración que un grupo de usuarios hacen de este producto, así como los elementos de la página que merecen mejor consideración. Así, las partes de Topics consideradas más útiles son: Navigator (por un $72 \%$ de encuestados), Articles about (67\%), Overview (64\%) y Headlines around the Web (59\%).

\section{Resultados}

Los resultados del análisis efectuado de la sección Times topics a partir de la observación y la entrevista a $\mathbf{O}^{\prime} \mathbf{N e i l}^{1}$ se desglosan en los 3 apartados de características: generales del servicio, de la página principal de la sección, y de las páginas de los temas.

\subsection{Características generales}

\subsubsection{Origen y objetivo de Times topics}

Nace con el cambio de diseño de la web del periódico realizado en 2006. En ese momento el objetivo inicial era hacer llegar al lector todo el material que utiliza un periodista para su artículo pero que se desecha cuando éste hace su trabajo de condensación. En la entrevista de Beckett (2011), O’Neil pone el ejemplo del periodista que trabaja en un tema semanas enteras y llega a recoger mucho material que se ve reducido a un artículo de 1.200 palabras: "Hay una gran cantidad de información que se ha obtenido durante este proceso que no llega al lector. La pregunta era: ¿cómo compartirla con él?"

Así nace Topics y va creciendo y adquiriendo características propias a medida que se van descubriendo y expandiendo las posibilidades de ofrecer información diversa agrupada por temas.

Finalmente, el servicio ha adquirido la forma de "una enciclopedia de acontecimientos actuales, al estilo de Wikipedia", y altamente fiable, ya que "nuestras páginas aportan cierto grado de autoridad: ofrecemos una reelaboración de lo que hemos editado con el nivel de calidad del New York times" (Beckett, 2011).

\subsubsection{Sistema de trabajo}

La sección cuenta con un equipo de trabajo de 5 personas: 3 editores (incluyendo a O'Neil) y 2 ayudantes. O'Neil describe así en nuestra entrevista la actividad de los integrantes del equipo: "Hasta cierto punto, todos hacemos lo mismo. Uno de los editores disecciona el periódico de papel cada mañana y crea una lista de topics que hay que crear o actualizar, y esto se da a conocer a todo el equipo. Los editores nos turnamos para leer todo el material impreso que se produce cada día. Los ayudantes suelen dedicarse a tareas más complicadas desde un punto de vista tecnológico y ayudan en el resto".

El trabajo de la sección sigue un curso paralelo al de la Redacción, ya que si los periodistas siguen un ciclo de 24 horas de producción de noticias, ellos recogen al día el material de referencia que generan los periodistas. Preguntado sobre la relación con el resto de la Redacción en la elaboración del contenido de Topics, O'Neil distingue la relación con los periodistas y con el equipo de documentación. De los periodistas proceden algunos de los textos de presentación o introducción de los temas, aunque "lo más frecuente es que nosotros escribamos las introducciones a partir de los artículos de un periodista y entonces se las pasamos para conocer su opinión". Y en cuanto a la relación con el equipo de documentación, señala que en los inicios de la sección, en los años 2006 y 2007, el servicio de documentación fue "de gran ayuda". En la actualidad, sin embargo, "por desgracia este departamento se ha reducido considerablemente, pero todavía les pedimos ayuda como por ejemplo para listas de fuentes importantes".

\subsubsection{Tipos de páginas}

Hay cuatro tipos de páginas agrupadas bajo los epígrafes: Personajes, Temas, Lugares y Organizaciones (People, Subjects, Places y Organizations). La primera categoría incluye personajes de todo ámbito y época; la segunda, páginas de materias de contenido diverso; en Places se agrupan las páginas de países y otros lugares destacados; y finalmente, Organizations incluye desde instituciones a partidos y desde oficinas de la Administración hasta empresas de todo el mundo. La manera de presentar la información al lector, sin embargo, es la misma en todas las páginas y no se ve modificada por esta distinción.

\subsubsection{Número de páginas}

A partir de la opción browser se han contabilizado todas las páginas de Topics. A 30 de diciembre de 2011 el servicio ofrece un total de 18.988 páginas. Por tipos se distribuyen de la siguiente manera (tabla 1): People, 9.731 páginas (51,2\%); Subjects, 2.116 (11,1\%); Places, 369 (1,9\%); Organizations, $6.772(35,7 \%)$. Destacan por tanto cuantitativamente las 
páginas dedicadas a personajes, que constituyen más de la mitad del total, seguidas por las Organizations, que son más de una tercera parte, mientras que los Subjects son algo más de una de cada diez páginas, y finalmente, la presencia de lugares es sensiblemente inferior, no llegando al $2 \%$.

\begin{tabular}{|l|r|r|}
\hline \multicolumn{1}{|c|}{ Tipo } & Número & $\%$ \\
\hline People & 9.731 & 51,3 \\
\hline Subjects & 2.116 & 11,1 \\
\hline Places & 369 & 1,9 \\
\hline Organizations & 6.772 & 35,7 \\
\hline Total & $\mathbf{1 8 . 9 8 8}$ & $\mathbf{1 0 0 , 0}$ \\
\hline
\end{tabular}

Tabla 1. Tipos y cantidad de páginas de Topics

En nuestra conversación con O’Neil, éste declaró que en la fase inicial de la sección de Topics, "la mayor parte de nuestras energías se dedicó a crear nuevas páginas, pero actualmente [...] sólo lo hacemos cuando surge un tema nuevo, o cuando algo o alguien que ha sido secundario pasa a ser importante para nuestro seguimiento. Esto da como resultado unas 20 ó 30 páginas nuevas al mes". También afirmó que estaban preparando "un proceso de consolidación que reducirá de manera significativa la cifra de 25.000 ", ya que algunas de estas páginas son "una mera indexación de resultados de búsquedas, sin ningún valor añadido por nosotros". Vemos que a 30 de diciembre de 2011, esta reducción se ha producido ya que el número total de páginas no llega a 19.000, y se han eliminado las páginas sin "valor añadido".

\subsubsection{Autoría}

En ningún caso aparece la autoría de la página de temas. La aportación de los cinco integrantes del equipo de Topics no aparece especificada ni se revela su autoría. Hay por tanto la intención de considerar como responsabilidad conjunta de la sección la totalidad del contenido presentado sin personalizar en ningún caso, reforzando así la idea de servicio y trabajo de equipo.

\subsubsection{Ayuda y contacto}

No existe en la página de presentación ningún enlace a páginas de ayuda a la navegación, la búsqueda o la consulta de contenidos del servicio, más allá de unas líneas de descripción propia de la página, aun cuando se puede considerar que la navegación es bastante intuitiva.

Tampoco se ofrece ninguna posibilidad de contacto específico con los responsables de la sección. Es un hecho negativo, que contrasta con la disponibilidad que sí tiene la mayoría de secciones de la edición digital del periódico, como por ejemplo Economía (Business), Información local (The Metro Desk), Internacional (The Foreign
Desk), Información nacional (The National Desk) o Deportes (Sports). El diario también permite contactar con los periodistas que firman los artículos, pero como las páginas de Topics no aparecen firmadas, tampoco hay posibilidad de contactar con sus editores.

\subsection{Página de sección}

\section{http://www.nytimes.com/pages/topics}

La página principal de la sección contiene 5 elementos o apartados diferenciados: el tema destacado (Featured topic), 6 temas de actualidad, 6 actualizados recientemente, un directorio o browser de la sección, y un breve texto de presentación. La estructura es de dos columnas: en la columna $A$ (izquierda, dos tercios de la página) los tres primeros, y en la columna B (derecha, un tercio de la página) los dos restantes. Se completa la página con espacio para publicidad (figura 1).

En la página hay muy poco texto y se da gran protagonismo a la imagen. El tema destacado se presenta siempre con una gran fotografía, del mismo ancho que la columna A. En cuanto a contenidos, sólo se muestran de manera directa 13 de las casi 19.000 páginas que contiene el producto.

A continuación se comenta cada uno de los elementos de la página:

\section{a. Featured topic}

Es el tema más destacado, que se presenta con un título y una gran fotografía. El enlace a la página del topic está tanto en el título como en la imagen. Es un tema amplio, que ofrece múltiples aspectos a tratar informativamente, y hay cierta tendencia a que sea de política internacional. Ejemplos son la guerra de Irak, el conflicto de Afganistán, o Corea del Norte, que se presentaba como Featured topic en diciembre de 2011 tras la muerte de Kim Jong II:

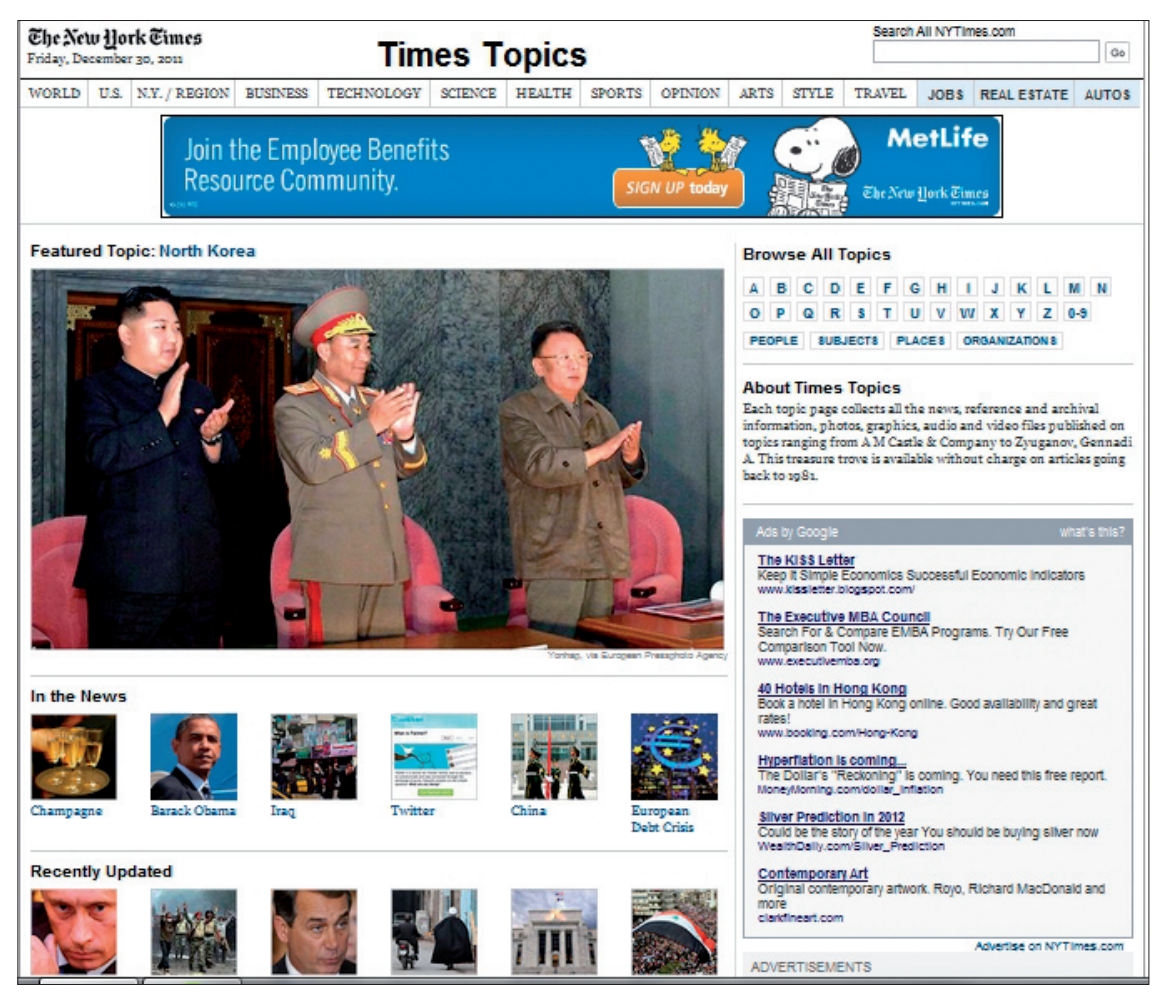

Figura 1. Página principal de Times topics el 30 de diciembre de 2011 
North Korea (figura 1)

http://topics.nytimes.com/top/news/international/coun triesandterritories/northkorea/index.htm/?ref=topics

\section{b. In the news}

Presenta 6 temas sin que sobresalga ninguno de ellos especialmente. Se muestra el título y una pequeña fotografía. El enlace a la página del topic está en el título y en la fotografía. No necesariamente están relacionados con noticias de actualidad. Por ejemplo, durante las Navidades de 2011 un tema fue el champán:

\section{Champagne wines}

http://topics.nytimes.com/top/reference/timestopics/sub jects/w/wines/champagne/index.htm/?ref=topics

\section{c. Recently updated}

También presenta 6 temas, que son los actualizados más recientemente. Como en el epígrafe anterior, sólo aparecen el título y una pequeña fotografía y el enlace a la página del tema está en el título y en la fotografía. A diferencia del anterior, estos temas sí guardan relación con la actualidad y si aparecen destacados es porque se han introducido novedades recientemente en sus páginas correspondientes. Un buen ejemplo que aparece a menudo en este apartado es la página sobre la situación de revueltas en Siria:

Syria protests (2011-).

http://topics.nytimes.com/top/news/international/coun triesandterritories/syria/index.htm/?ref=topics

\section{d. Browse all topics}

Es un apartado interesante desde el punto de vista del acceso al conjunto de la información de la sección. No incluye un buscador, como sería deseable, sino únicamente browsing con dos opciones:

a) Listado alfabético de 27 entradas correspondientes a las 26 letras del abecedario más una entrada numérica (1-9). Accediendo a cada entrada se muestra el listado ordenado alfabéticamente de los temas de la letra correspondiente, sin distinción de los cuatro tipos de páginas existentes.

b) Clasificación de las páginas en las 4 categorías ya señaladas de personajes, lugares, organizaciones y temas. Dentro de cada categoría, la presentación también es alfabética.

\section{e. About Times topics}

Presentación de la página, con esta descripción: Each topic page collects all the news, reference and archival information, photos, graphics, audio and video files published on topics ranging from A $M$ Castle \& Company to Zyuganov, Gennadi A. This treasure trove is available without charge on articles going back to $1981^{2}$. Como se ha señalado anteriormente, se echa en falta un texto explicativo más detallado sobre los contenidos de la sección y el sistema de acceso a los mismos.

\subsection{Página del Topic}

Las páginas de los temas se presentan en tres columnas: la primera y la tercera a $2 / 5$ de la página, y la central, a $1 / 5$ de página. Hay una serie de elementos que aparecen mayoritariamente en todas las páginas, independientemente de su tipo: imagen, presentación, artículos, buscador, blogs, multimedia, Headlines around the web, Navigator, enlaces a otras noticias u otros topics, servicio de alerta y rss, y publicidad. La distribución se realiza en la forma que se muestra en la tabla 2:

\begin{tabular}{|c|c|c|}
\hline Columna A & Columna B & Columna C \\
\hline $\begin{array}{l}\text { a. Ilustración } \\
\text { b. Presentación } \\
\text { c. Articles about } \\
\text { d. Buscador }\end{array}$ & $\begin{array}{l}\text { e. Blogs } \\
\text { f. Multimedia } \\
\text { g. Headlines around the web } \\
\text { h. Navigator }\end{array}$ & $\begin{array}{l}\text { [Publicidad] } \\
\text { i. Related topics } \\
\text { j. Most popular, rss, } \\
\text { alertas }\end{array}$ \\
\hline
\end{tabular}

Tabla 2. Estructura más habitual de una página de Topic

La estructura se repite en la mayoría de páginas, con algunas variaciones: por ejemplo, las de países pueden presentar una pequeña ficha descriptiva del país y un mapa, o algunos temas menores pueden ofrecer simplemente noticias clasificadas por fecha y por etiquetas, en algunos casos con una breve introducción, en otros sin la misma.

O’Neil explica que "en la mayoría de casos seguimos una estructura para dar coherencia a las páginas, pero modificamos el modelo cuando nos parece necesario". Por tanto, se puede señalar a partir de la observación realizada y las declaraciones de O'Neil, que la estructura se plantea de una manera flexible, y que se puede modificar en función del criterio de los editores.

A continuación se comentan los diferentes elementos constitutivos de las páginas de Topics.

\subsubsection{Elementos principales}

\section{a. Ilustración}

En los temas de mayor actualidad suele encabezar la columna izquierda una ilustración. Generalmente es una fotografía, pero puede ser un mapa si la página es sobre un país. En los temas menores no hay ilustración.

\section{b. Presentación o introducción al tema}

Es un elemento importante dentro del conjunto de contenidos del topic ya que es el primer texto de la página y el más destacado. Consiste en una presentación actualizada del tema, que en ocasiones puede llegar a ser muy detallada (oscila entre 500 y 4.000 palabras). La redacción del mismo no siempre corresponde al equipo de la sección Topics. O’Neil explica el funcionamiento habitual: "A veces algún redactor escribe a partir de cero una introducción para nosotros. Aunque lo más frecuente es que nosotros escribamos las introducciones a partir de los artículos de un periodista y luego se las pasemos para conocer su opinión".

Este texto no tiene un título propio para cada topic, y el encabezamiento es Overview (visión general), o bien, Recent developments y Overview (acontecimientos recientes y visión general). En estos últimos casos se trata de temas de más actualidad y que están en permanente actualización, y se presentan siguiendo el esquema siguiente:

- Recent developments. Una breve explicación (a veces cronológica) de los acontecimientos más recientes. 
- Overview. Texto con la visión de conjunto del tema en cuestión.

Ejemplo: Topic sobre la crisis de la deuda europea:

European debt crisis

http://topics.nytimes.com/top/reference/timestopics/sub jects/e/european_sovereign_debt_crisis/index.htm/? ref=topics

El modelo -comenta O'Neil- es: “unas introducciones que den una breve visión general de cada tema, seguidas de una cronología que ofrezca una mayor profundidad. Pero cuando un tema es de tanta actualidad que sería difícil para el lector encontrar la información más reciente, probamos con una cronología (Recent developments) que resuma los principales acontecimientos (por ejemplo, la crisis nuclear de Japón tras el tsunami o la crisis en Libia)"3.

\section{c. Articles about}

Selección realizada por el equipo de Topics de los artículos de The New York times relacionados con el tema, ordenados por fecha de más reciente a más antiguo (con la opción de ordenarlos al revés), de los que se muestra: titular, una breve descripción, en algunos una pequeña foto y una serie de tags (etiquetas) o descriptores.

Por ejemplo, el topic de Siria presenta 4.100 artículos desde 1980. Lo más interesante es que el clic en cada etiqueta ejecuta la siguiente búsqueda: tema del topic + descriptor, con lo cual se ofrece la posibilidad de recuperar todos los artículos del tema bajo las mismas etiquetas.

Hay que destacar que los artículos de este apartado son el resultado de una selección realizada por los profesionales de la sección a partir de un proceso de búsqueda. En nuestra entrevista, O'Neil describe así el procedimiento: "Los artículos sobre cada topic se muestran en un listado por orden cronológico; este proceso es automatizado y está elaborado a partir de un buscador que busca entre las palabras destacadas de los índices. A veces la búsqueda es sencilla, como por ejemplo en 'Lincoln, Abraham', en la que hay un término que encaja perfectamente, pero otras veces nuestros taxonomistas tienen que combinar una serie de términos y ajustar el resultado para descartar artículos irrelevantes".

\section{e. Blogs propios}

Blogs del propio periódico especializados en el tema. Por ejemplo, en la página de Barack Obama se destaca el blog "The caucus", de análisis de la política y del Gobierno norteamericano.

\section{Barack Obama}

http://topics.nytimes.com/top/reference/timestopics/peo ple/o/barack_obama/index.htm/?ref=topic

The caucus

http://thecaucus.blogs.nytimes.com

\section{f. Galería multimedia}

En este apartado aparecen diversos recursos, pero siempre de tres tipos: gráficos (estáticos o interactivos), vídeos y galerías de fotos. La diversidad es considerable: hay páginas con uno, con dos o con los tres tipos de formatos multimedia, y a su vez, pueden aparecer desordenados y no siempre agrupados conjuntamente. En este sentido se aprecia falta de sistematización. Es un apartado interesante para el usuario, ya que se nutre del prestigioso departamento multimedia del diario, que produce algunos de los gráficos interactivos de mayor calidad de la Web.

\section{Headlines around the web y Navigator son dos excelentes ejemplos de "perio- dismo de enlaces" con una selección de las mejores fuentes sobre el topic}

\section{g. Headlines around the Web}

Se trata de enlaces a noticias de actualidad de otros medios digitales, de aspectos recientes y que se renuevan frecuentemente; por lo tanto, están vinculados a la información más inmediata.

Es muy interesante la presencia de este apartado, que constituye un excelente ejemplo de "periodismo de enlaces": el diario The New York times está ofreciendo a sus lectores las mejores informaciones no ya propias sino de otros medios digitales, algo poco extendido en el periodismo digital.

\section{d. Buscador}

Al final de la lista anterior de artículos hay un buscador que permite buscar por palabras clave entre los artículos archivados que versan sobre el tema en cuestión. Las opciones de búsqueda son tres: match any word, match all words y match exact phrase, es decir, las equivalentes respectivamente a los operadores booleanos OR y AND, y la búsqueda por frase exacta.

Paul, Hansen y Suzuki (2011) señalan acertadamente que un aspecto negativo del servicio Topics es que, si bien existe esta opción de búsqueda, al estar al final de la página es fácil que pase desapercibida a algunos usuarios.

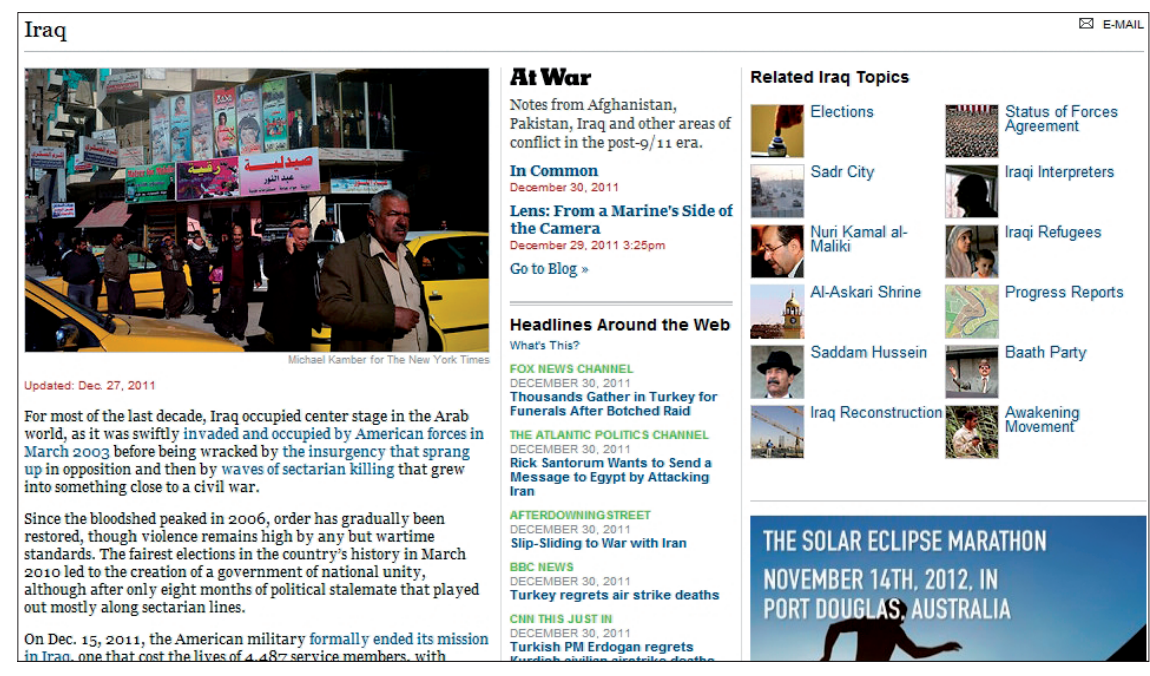

Figura 2. Topic de Iraq con los Headlines around the web 
Por ejemplo, en un tema de actualidad como Irak, el 30 de diciembre de 2011 se ofrecían enlaces a noticias del día de las cadenas Fox, $B B C$ y $C N N$ y de las webs The Atlantic politics channel y After Downing street (figura 2).

\section{h. Navigator}

Enlaces a webs de medios, entidades o instituciones relacionadas con el tema, como especiales o temas de prensa digital, documentos oficiales, libros, artículos, informes, etc. Es otro apartado dedicado al "periodismo de enlaces" que complementa al anterior, ya que aquí hay fuentes de todo tipo y en el anterior exclusivamente noticias.

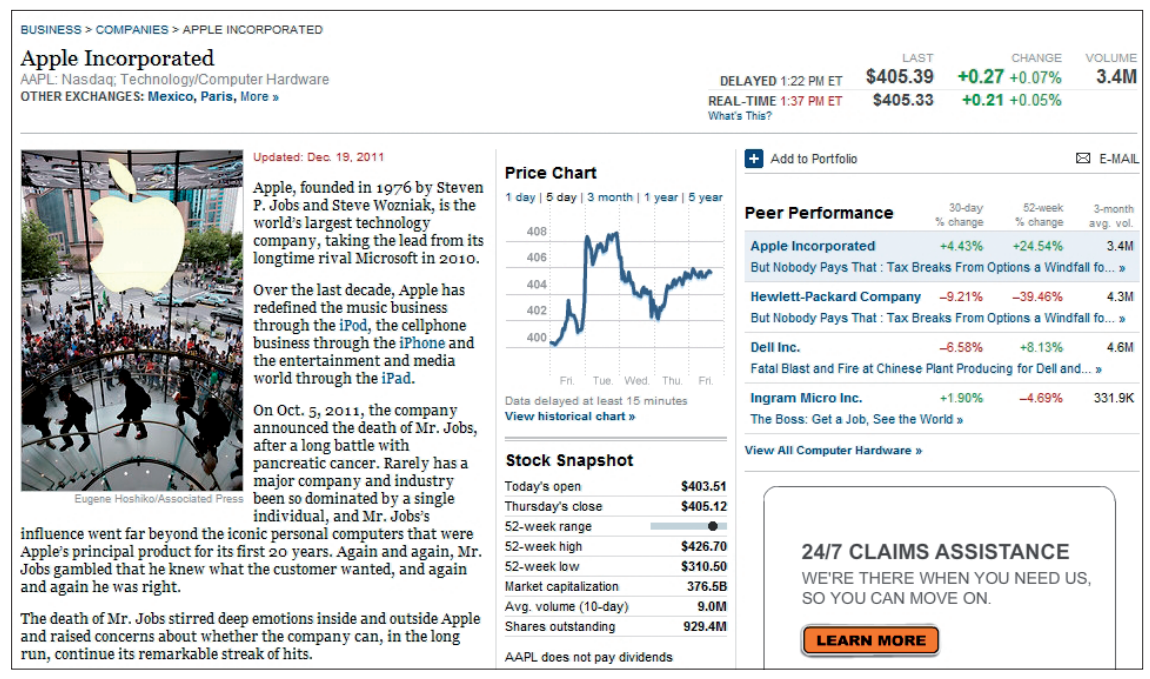

Por ejemplo, en la misma página de Irak antes mencionada, Navigator ofrece enlaces entre otros a los siguientes recursos web sobre el tema:

- Departamento de Defensa http://www.defense.gov/news

- Iraq Bodycount, entidad británica que trata de contabilizar las víctimas civiles del conflicto http://www.iraqbodycount.org

- lista de recursos sobre Irak de la biblioteca del City College de Nueva York

http://www.ccny.cuny.edu/library/Divisions/Govern ment/Iraqbib.html

- página oficial del Gobierno iraquí (en árabe) http://www.cabinet.iq

- etc.

\section{i. Related topics}

No aparece en todos los temas, solamente en aquellos más importantes o más desarrollados (véase en la figura 2, Related topics de Irak). Se trata, como su nombre indica, de una selección de otras páginas de Topics relacionadas.

\section{j. Most popular, rss, alertas.}

Por último, en la columna derecha se muestran las noticias más populares del web divididas en las categorías e-mailed, blogged, searched y dos servicios clave para que el usuario pueda recibir información actualizada y personalizada sobre ese tema, como son la suscripción rss y la recepción de alertas.

\subsubsection{Particularidades}

Además de esta estructura mayoritaria, algunas particularidades destacables que se pueden señalar son las de las páginas de empresas y de periodistas. Las primeras están incluidas en la categoría de las organizaciones, y además de lo ya señalado ofrecen otros tipos de información:

a. Company information. Breve ficha descriptiva de la empresa, con la fecha de fundación, actividad, sector, productos, etc. (en la columna A).

b. Principales cifras económicas. Por ejemplo, cotización en bolsa, beneficios por acción, beneficios por trimestre, nombres de los principales ejecutivos, etc. (columna B).

c. Peer performance. Se informa de su actuación en la bolsa en comparación con empresas del mismo sector (columna C).

Un buen ejemplo es la página de la empresa Apple.

http://topics.nytimes.com/topics/news/business/compa nies/apple_computer_inc/index.html

Por último hay que señalar que los periodistas de The New York times tienen su propia página en la sección Times topics, en la que se ofrecen los artículos publicados por ellos. Se accede a estas páginas clicando en el nombre del periodista que encabeza los artículos. Una vez en la página, además de acceder a todos los artículos del reportero en cuestión, un enlace lleva a su dirección de correo. En algunos hay un breve perfil.

Ejemplo: página en Topics del periodista James Risen http://topics.nytimes.com/topics/reference/timestopics/ people/r/james_risen/index.html

\section{Conclusiones}

Times topics es un modelo de referencia de producto periodístico documental en cuanto que, más allá de las noticias del día a día, sitúa las informaciones periodísticas en un contexto temporal más amplio para mostrar en profundidad un tema (personaje, país, organización o propiamente tema) explotando la variedad de características propias del periodismo digital ${ }^{4}$ (principalmente multimedia, hipertextualidad y documentación, y menos la interactividad) y acudiendo tanto a fuentes de información digitales del propio diario (Articles about, Overview, buscador...) como externas (Headlines around the web y Navigator).

El trabajo periodístico-documental que llevan a cabo los editores de Times topics se puede considerar una excelente muestra en el terreno del periodismo de lo que en la actualidad se denomina content curation: se trata de seleccionar, ordenar, elaborar y presentar al usuario la mejor información en la Web sobre un tema. El resultado de una página de topics es eso precisamente: una selección de la información sobre el tema en cuestión publicada por el propio diario junto a la considerada como más relevante por los editores (content curators) de la sección Topics de entre la existente 
en la Web. Una selección que se elabora, se jerarquiza y se complementa con textos introductorios, y servicios como el buscador o la suscripción a actualizaciones mediante rss y alertas.

Es por ello una fuente de información de notable valor para usuarios diversos, además del público generalista: profesionales del periodismo y la documentación, estudiantes, docentes, investigadores... Codina (2010, p. 153) argumenta que algunos sitios web de empresas de comunicación pueden considerarse en estos momentos la "cumbre de la Web" y cita, entre otros cibermedios The New York times. Siguiendo esta argumentación, podemos considerar asimismo la sección de Times topics una de las cumbres de la documentación periodística en internet.

Times topics es una excelente muestra de content curation: se trata de seleccionar, ordenar, elaborar y presentar al usuario la mejor información en la Web sobre un tema

Por otra parte, se pueden reseñar algunos aspectos a mejorar. En la página de sección se ofrece la posibilidad al lector de accesos alfabético y temático a los topics (Browse all topics), pero se echa en falta la existencia de un buscador de temas, que sí está presente en la página de cada topic para buscar artículos de ese tema (aun cuando su ubicación sea mejorable). En segundo lugar, se puede señalar la falta de autoría de los diferentes elementos de la página (desde el artículo central de Overview a las diferentes selecciones de recursos). Y por último, y de manera especial, hay que decir que la sección presenta una baja interactividad (es la característica del periodismo digital menos desarrollada en la sección Topics). Ésta no existe más allá del nivel mínimo de interactividad que representa la navegación web, o de la que ya tenían los elementos originales incorporados (artículos, gráficos multimedia, etc.). Ya no se trata solamente de la ausencia de ayudas al usuario o de la imposibilidad por parte de éste de contactar con la sección en conjunto o con los editores individualmente, sino que además hay que añadir la ausencia de opciones de realizar comentarios por parte de los lectores o de interactuar o compartir los contenidos de Topics en medios sociales, algo poco comprensible en la actual época de la web social.

Para terminar, parece oportuno dejar constancia de una reflexión que se desprende del método de trabajo del equipo de Topics: durante nuestra conversación John O'Neil contó que las páginas se crean o se actualizan a partir de "diseccionar cada mañana el periódico de papel". Sin que de ello haya que sacar una conclusión definitiva, el hecho de que un producto característico del periodismo digital como la sección de Topics del New York times se actualice "en función" de lo que aparece publicado en su versión impresa indica que para los directivos del diario la jerarquía informativa del papel todavía mantiene (en 2011) su papel de referencia frente a la continua actualización de la web.

\section{Notas}

1. Todas las declaraciones atribuidas a $\mathbf{O}^{\prime}$ Neil en este artículo son resultado de la entrevista que nos concedió, con la única excepción de las referencias del apartado 4.1.1 Origen y objetivo de Times topics, que corresponden a la entrevista de Beckett (2011), como así se indica. Cuando preguntamos a O'Neil sobre el origen de Topics, nos remitió a esa entrevista anterior. El resto de declaraciones incluidas en este trabajo (apartados: 4.1.2 Sistema de trabajo; 4.1.4 Número de páginas; 4.3 Página del Topic; 4.3.1 Elementos principales; y 5 Conclusiones) son originales de este artículo.

2. "Cada página de cada tema recoge todas las noticias e información de referencia y de archivo, así como fotos, gráficos y documentos de vídeo y de audio publicados sobre temas que van desde A M Castle \& Company a Zyuganov, Gennadi A. Un tesoro gratuito con artículos publicados desde 1981".

3. Japan-earthquake, tsunami and nuclear crisis (2011). http://topics.nytimes.com/top/news/international/coun triesandterritories/japan/index.htm/?scp=6\&sq=Fukushima \&st $=$ cse

Libya - Revolution and aftermath.

http://topics.nytimes.com/topics/news/international/coun triesandterritories/libya/index.html

4. Véanse por ejemplo estas características en Abadal; Guallar, 2010, pp. 40-46.

\section{Bibliografía}

Abadal, Ernest; Guallar, Javier. Prensa digital y bibliotecas. Gijón: Trea, 2010. ISBN 9788497044462.

Albornoz, Luis. Periodismo digital. Los grandes diarios en la Red. Buenos Aires: Ediciones La Crujía, 2006, ISBN 978 9876010160.

Beckett, Lois. "The context-based news cycle: editor John O'Neil on the future of The New York Times' Topics Pages". Nieman Journalism Lab, February 23, 2011.

http://www.niemanlab.org/2011/02/the-context-basednews-cycle-editor-john-oneil-on-the-future-of-the-newyork-times-topics-pages/

Codina, Lluís. "El periodismo digital, lo mejor que tenemos en la Web". En: Abadal, Ernest; Guallar, Javier. Prensa digital y bibliotecas. Gijón: Trea, 2010, pp. 151-154.

Domingo, David. "Rutinas profesionales y valores en las redacciones de medios digitales catalanes: periodismo digital en contextos reales". II Congreso Online del Observatorio de la Cibersociedad, 2005.

http://www.cibersociedad.net/public/documents/89 4q5b.rtf

Guallar, Javier. "Documentación fotográfica en la prensa. Casos de El país, El periódico y La vanguardia". El profesional de la información, 2011a, v. 20, n. 4, pp. 392-398.

http://dx.doi.org/10.3145/epi.2011.jul.05

Guallar, Javier. "La documentación en la prensa digital. Nuevas tendencias y perspectivas". III Congreso intl de ciberperiodismo y web 2.0, Bilbao, 2011b, 9-11 noviembre. Universidad del País Vasco.

http://hdl.handle.net/10760/16326 
Guallar, Javier; Rovira, Cristòfol; Ruiz, Sara. "Multimedialidad en la prensa digital. Elementos multimedia y sistemas de recuperación en los principales diarios españoles". El profesional de la información, 2010, v. 19, n. 6, pp. 620-629. http://eprints.rclis.org/bitstream/10760/15088/1/620-631Guallar-Rovira-Ruiz.pdf http://dx.doi.org/10.3145/epi.2010.nov.08

Larrondo-Ureta, Ainara. El reportaje hipermedia. Análisis del género en los especiales de EIMundo.com, EIPaís.com y LaVanguardia.es. Tesis doctoral. Universidad del País Vasco. 2007.

Larrondo-Ureta, Ainara. "Estrategias de producción online para el tratamiento informativo en profundidad". Revista latina de comunicación social, 2008, n. 63, pp. 473-479. http://www.ull.es/publicaciones/latina/08/40_797_63_Bil bao/Ainara_Larrondo_Ureta.html

López-Aguirre, José-Luis. "Estudio de servicios y productos documentales en la prensa digital mexicana". Documentación de las ciencias de la información, 2009, v. 32, p. 93132.

http://www.ucm.es/BUCM/revistas/inf/02104210/articu los/DCIN0909110093A.PDF

Martínez-Rubio, Rosa. "La recuperación de la información en los periódicos digitales valencianos". En: López García, Guillermo. Comunicación local y nuevos formatos periodís- ticos en internet: cibermedios, confidenciales y weblogs. Valencia: Servei de Publicacions de la Universitat de València, 2008.

http://www.cibermediosvalencianos.es/comloc/Martinez.pdf

Nuño, María-Victoria. "Organización de los web site de periódicos españoles en internet". Hipertext.net, 2003, n. 1. http://www.hipertext.net

Paul, Nora; Hansen, Kathleen A.; Suzuki, Yoshi. "Curation questions and the start of some answers". OJR: The online journalism review, February 6, 2011.

http://www.ojr.org/ojr/people/paulhansensuzuki/ 201102/1939/

Rubio-Lacoba, María. Documentación informativa en el periodismo digital. Madrid: Síntesis, 2007 ISBN 978 8497564595

Rubio-Lacoba, María; Blanco-García, Juan-Carlos. "Mejor que un buscador, un encontrador". Documentación de las ciencias de la información, 2010, n. 33, pp. 273-287. http://hdl.handle.net/10760/16306

Sánchez-Vigil, Juan-Miguel; Marcos-Recio, Juan-Carlos; Olivera-Zaldua, María. "Influencia de la fotografía digital en los departamentos de documentación de prensa". El profesional de la información, 2009, v. 18, n. 3, pp. 278-283. http://dx.doi.org/10.3145/epi.2009.may.04

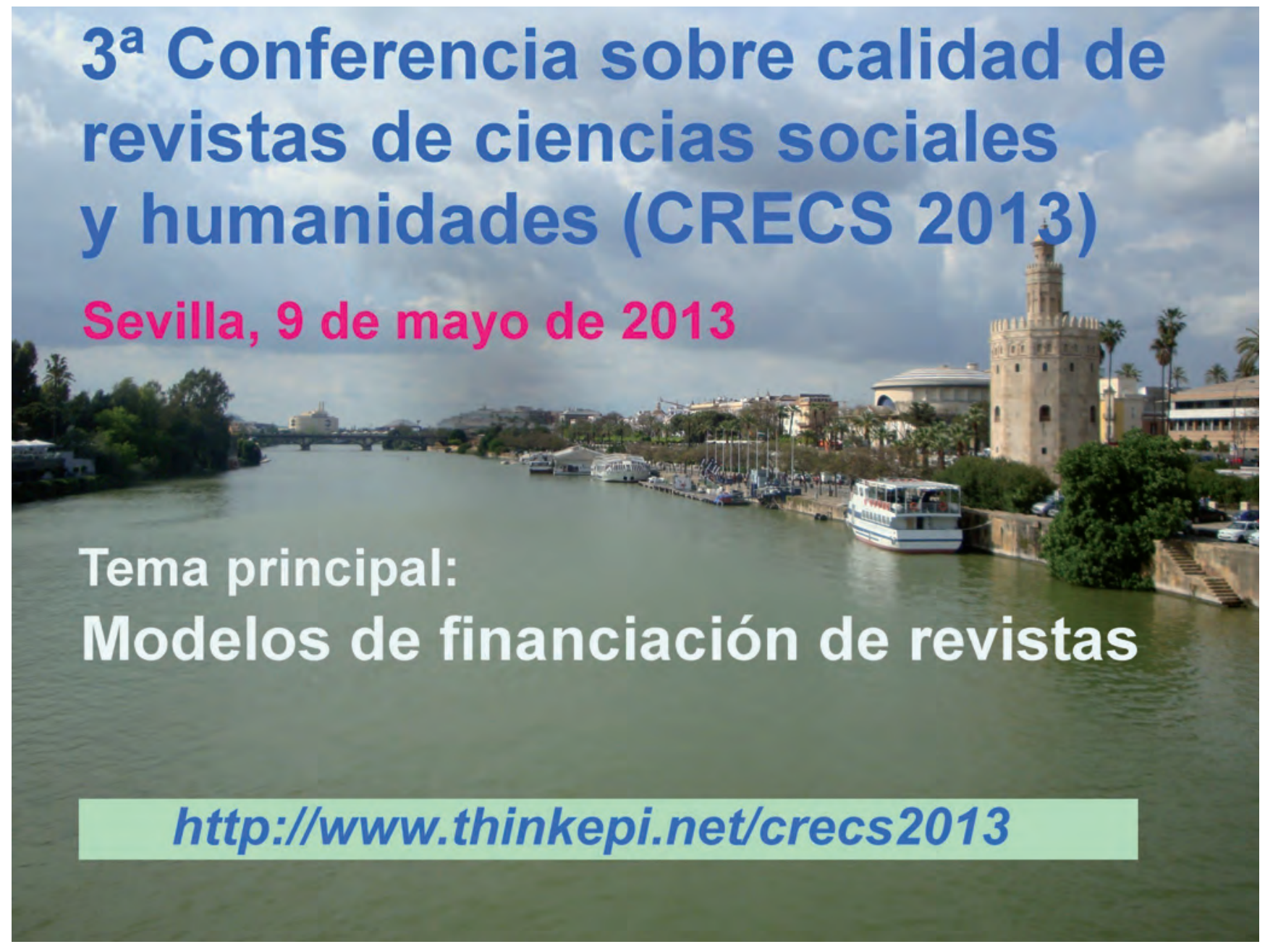

